\title{
Explantación y reposición de implante con dehiscencia vestibular en la zona estética. Reporte de caso.
}

\section{Explantation and replacement of an implant with a facial dehiscence in the aesthetic region. Case report.}

\author{
Sebastian Vilugron ${ }^{1}$, Pablo Cifuentes ${ }^{2}$, Natalia Marcus ${ }^{3}$, Blas Galdames ${ }^{3}$, Michael Wendler ${ }^{3 *}$
}

\author{
1. Programa de Implantología con Mención en \\ Reconstrucción, Protéstica y Estéticamente \\ Guiada, Facultad de Odontología, Universidad de \\ Concepción, Chile. \\ 2. CDC Dental, Concepción, Chile. \\ 3. Departamento de Odontología Restauradora, \\ Facultad de Odontología, Universidad de \\ Concepción, Chile. \\ * Correspondencia Autor: Dr. Michael Wendler \\ - Departamento de Odontología Restauradora, \\ Facultad de Odontología, Universidad de \\ Concepción | Dirección: Roosevelt 1550 \\ Concepción, Chile |E-mail: mwendler@udec.cl | \\ Teléfono: +56412661786 - +56412204481 \\ Trabajo recibido el 17/12/2019. \\ Aprobado para su publicación el 28/06/2020
}

\begin{abstract}
RESUMEN
La incidencia de complicaciones en los tejidos periimplantarios, como recesiones y dehiscencias, ha ido en aumento en los últimos años, principalmente asociados a un incorrecto posicionamiento espacial de los implantes. El objetivo de este reporte de caso es presentar el manejo quirúrgico de una complicación estética debida a la mal posición de un implante en la zona anterior. Caso. Paciente se presenta con recesión mucosa y dehiscencia por vestibular del implante 1.2 , causados por su mal posicionamiento. Se realiza explantación mediante llave de alto torque e inserción de un nuevo implante en combinación con regeneración ósea (sticky bone) e injerto de tejido conectivo, lo que recupera la armonía gingival. Conclusión. La explantación conservadora acompañada de regeneración tisular ofrecen una interesante alternativa para el tratamiento de defectos estéticos severos asociados a la mal posición de implantes. La sistematización de este tipo de protocolos es fundamental para mejorar su predictibilidad.
\end{abstract}

PALABRAS CLAVE:

Implante dental; Dehiscencia; Explantación; Llave de alto torque.

Int. J. Inter. Dent Vol. 13(3); 161-164, 2020.

\section{ABSTRACT}

An increase in the incidence of peri-implant soft tissue complications, such as facial recession and dehiscence, has been observed in the last years, mainly associated with an incorrect spatial placement of the implants. This case report focuses on the surgical management of an esthetic complication due to an incorrect implant position in the anterior region. Case report. Patient presented with recession and dehiscence in the facial area of implant 1.2, due to its incorrect placement. Explantation was performed with a high torque wrench, followed by the immediate placement of a new implant in combination with bone regeneration (sticky bone) and soft tissue augmentation. Conclusion. The use of atraumatic explantation techniques followed by guided tissue regeneration offers an interesting alternative for the treatment of severe defects in the esthetic region due to incorrectly placed implants. An adequate systematization of these protocols is key to improve their predictability.

KEY WORDS:

Dental implant; Dehiscence; Explantation; High torque wrench.

Int. J. Inter. Dent Vol. 13(3); 161-164, 2020.

\section{INTRODUCCIÓN}

La instalación exitosa de implantes en el sector anterior es uno de los grandes desafíos de la implantología moderna, ya que para lograr una rehabilitación estética y funcional hay que respetar una serie de parámetros biológicos, quirúrgicos y protésicos. Es por ello que la definición de éxito en la terapia con implantes no depende únicamente de su supervivencia a largo plazo, sino también de su funcionalidad, estética, estabilidad tisular ${ }^{(1)}$, así como de la satisfacción del paciente(2).

Actualmente se observa un aumento cada vez mayor en la incidencia de fracasos estéticos en implantología, generalmente asociados a una mala planificación, falta de conocimiento y experiencia del cirujano ${ }^{(3)}$. Antiguamente el concepto para la instalación de un implante se basaba en la disponibilidad ósea, siguiendo la dirección del hueso remanente, lo que frecuentemente conducía a errores en la posición espacial del implante con respecto a la futura restauración ${ }^{(3)}$. Esto, a su vez, generaba dificultades técnicas y complicaciones biológicas ${ }^{(4)}$, siendo la dehiscencia de tejidos blandos periimplantarios en la zona estética una de las más complejas de tratar ${ }^{(5)}$. En este sentido, el posicionamiento vestibularizado de la plataforma protésica ha sido asociado a un riesgo hasta tres veces más elevado de desarrollar dehiscencias en los tejidos periimplantarios ${ }^{(6)}$. Por lo tanto, la colocación espacial ideal del implante resulta un factor clave para lograr un resultado estético y prevenir complicaciones ${ }^{(7,8)}$.

La solución de estos casos resulta compleja y poco predecible, ya que generalmente depende de la experiencia y destreza del cirujano. En la literatura se han descrito distintos enfoques y tratamientos para enfrentar el manejo de los tejidos blandos en implantes con problemas estéticos menores debidos a su malposición, y que por lo tanto no justificarían su remoción ${ }^{(5,7,9-10)}$. En paralelo, una mal posición leve a moderada se puede resolver protésicamente mediante una correcta selección de los aditamentos, así como a través de la personalización de los pilares, otorgando resultados estéticamente aceptables. Sin embargo, los casos 
de mal posición severa no dejan margen y requieren un tratamiento radical, a través de la remoción (explantación) del implante ${ }^{(1)}$.

Las técnicas de explantación usualmente son invasivas, lo que conlleva el riesgo de complicaciones y pérdida ósea, volviendo más compleja la resolución del caso e implicando la necesidad de procedimientos adicionales ${ }^{(4)}$. Existen diversas técnicas para la explantación, dentro de las cuales las más utilizadas son la remoción mediante trefina y la desinserción del implante mediante llave de alto torque ${ }^{(11)}$. Sin embargo, el procedimiento de remoción de implantes mediante trefina constituye una técnica más invasiva e impredecible, lo que implica necesariamente una pérdida de hueso periimplantar. Es por ello que el uso de la llave de alto torque es el tratamiento de primera elección en muchas situaciones clínicas, debido a la mayor conservación de hueso y a la predictibilidad que esto permite para la inserción de un nuevo implante en la misma zona $^{(11)}$

El objetivo del presente reporte de caso es describir el abordaje de una complicación estética severa producto de un implante mal posicionado mediante su explantación con llave de alto torque y su sustitución por un implante con un adecuado posicionamiento protésico. Dada la complejidad estética del caso, fue necesario además utilizar un injerto combinado de hueso y tejido conectivo en la zona para corregir la dehiscencia causada por el tratamiento anterior.

\section{INFORME DEL CASO}

Paciente de género femenino, sin antecedentes mórbidos de relevancia, consulta por problemas estéticos en el sector anterior, principalmente a nivel de incisivos laterales. Al examen clínico se observa desarmonía gingival evidente y coronas clínicas alargadas hacia cervical en dientes 1.2 y 2.2. En ambos casos se presentan prótesis fijas metal cerámicas sobre implantes de conexión hexagonal externa, con una excesiva vestibularización de la plataforma, siendo más crítico en el incisivo lateral derecho (Figura 1), que además presentaba una dehiscencia importante en la zona. Si bien la paciente busca un tratamiento integral de la zona estética, por motivos económicos se realizó en esta etapa sólo el tratamiento en la zona del diente 1.2.

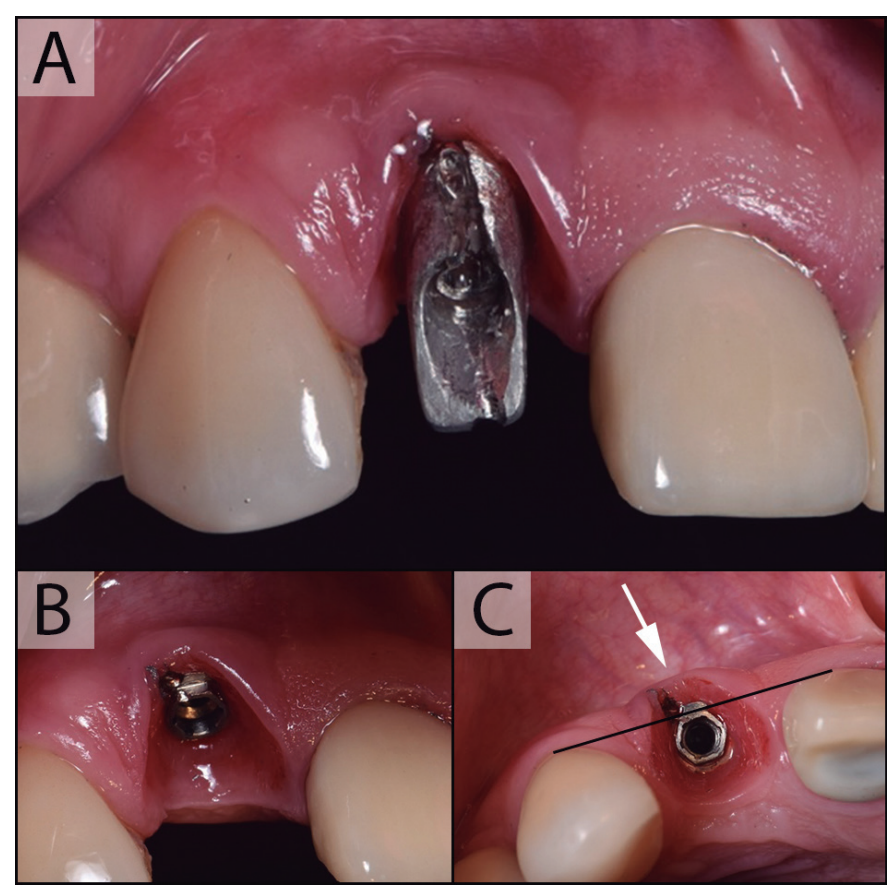

Figura 1. Retiro de prótesis fija implantosoportada en zona del diente 1.2. Este es un procedimiento que debe realizarse con sumo cuidado, para no dañar el tornillo protésico, la plataforma del implante y/o los tejidos blandos periimplantarios. En A se evidencia la posición alta del margen gingival con respecto a piezas vecinas, lo que se acompaña de dehiscencia del tejido subyacente. En B se observa la conexión hexagonal externa del implante, dañada durante la remoción de la prótesis fija. En C se puede visualizar la posición vestibularizada de la plataforma del implante con respecto a las piezas vecinas. Esto llevó a una posición vestibulizada de la prótesis fija, como se ve confirmado por el perfil de emergencia de la misma (flecha blanca).
El plan de tratamiento consideró la explantación del implante en 1.2 y la instalación en su lugar de un implante de conexión cónica interna de $3.5 \times 13 \mathrm{~mm}$ (Neobiotech, Seúl, Corea del Sur), así como la utilización de un aloinjerto óseo y un injerto de tejido conectivo palatino para manejar la dehiscencia vestibular.

Previo a la cirugía se extrajo sangre con el objetivo de obtener fibrina rica en factores de crecimiento y se mezcló con las partículas del aloinjerto (MinerOss Cortical ${ }^{\circledR}$, Biohorizons ${ }^{\circledR}$, Nashville, USA), para su utilización en la denominada técnica de "sticky bone" de Sohn y cols. ${ }^{(12)}$. La explantación se realizó mediante la técnica de llave de alto torque (Figura 2) utilizando el Neo Fixture Remover Kit (Neobiotech, Seúl, Corea del Sur). Posteriormente se realizó un colgajo tipo sobre de espesor parcial con tunelización de papilas mesial y distal a la altura de sus bases (Figura 3A). Previo a la instalación del implante se realizó la toma del injerto de tejido conectivo desde el paladar (Figura 3B). Se logró un torque inicial del implante por sobre $35 \mathrm{~N} / \mathrm{cm}$ (medido por el motor quirúrgico iChiropro, Bien air, Bienne, Suiza), lo que posibilitó su provisionalización inmediata. A continuación se fijó el injerto conectivo (Figura 4A y B) y se procedió al posicionamiento del sticky bone (Figura $4 C)$, dejándolo por debajo del tejido conectivo injertado. El provisional se ajustó al pilar provisorio, dejándolo sin contacto oclusal, tanto en céntrica como en excéntrica (Figura 5A). Se realizó un control a los 14 días para el retiro de suturas y se esperó un tiempo de 6 meses para permitir una adecuada oseointegración y estabilización de los tejidos injertados (Figura 5B). Previo a la rehabilitación final se solicitó un cone beam de control (Figura 6). La prótesis fija definitiva se realizó utilizando un pilar recto de titanio (ISAH415, Neobiotech) y una estructura de disilicato de litio LTA1 (e.max CAD, Ivoclar-Vivadent, Schaan, Lichtenstein) para conformar un pilar híbrido y posteriormente una corona monolítica del mismo material cementada (Figura 7).

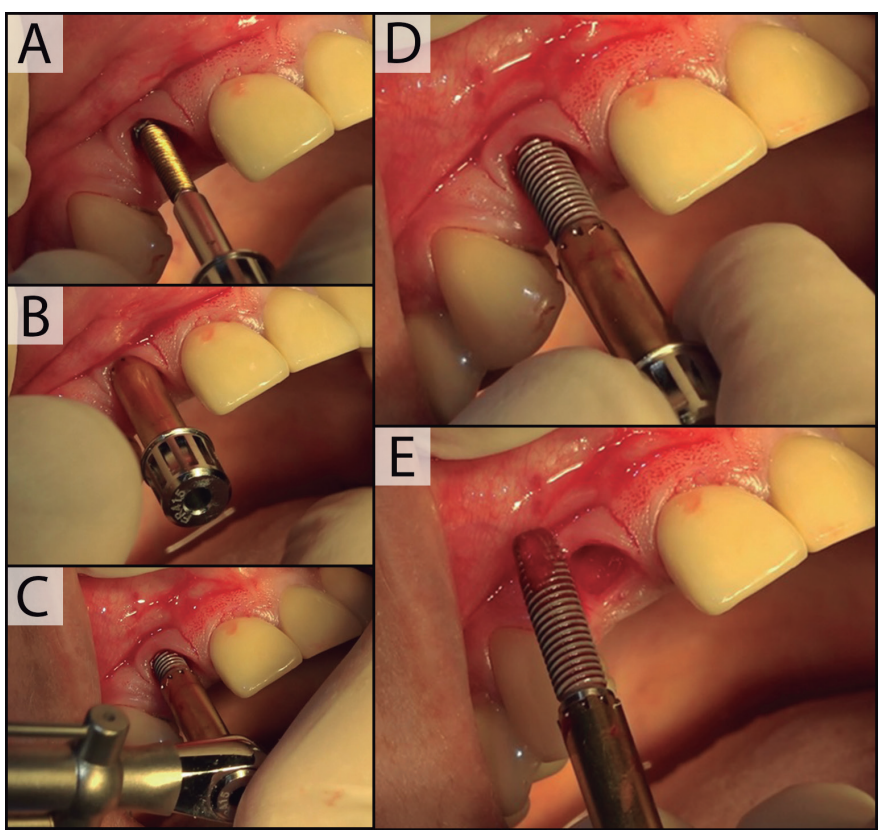

Figura 2. Secuencia de explantación mediante la técnica de la llave de alto torque. Ésta alcanza los $500 \mathrm{~N} / \mathrm{cm}$. A conexión del extractor a la plataforma del implante en sentido horario y se torquea a $50 \mathrm{~N} / \mathrm{cm}$. B inserción del removedor de implantes en sentido anti horario. C ajuste del ratchet y comienzo de la explantación en sentido antihorario. $\mathrm{D}$ remoción manual posterior a la ruptura de la oseointegración. E se observa el implante limpio, sin hueso adherido a su superficie, y el alveolo con sus paredes óseas y tejidos periimplantarios totalmente conservados. 


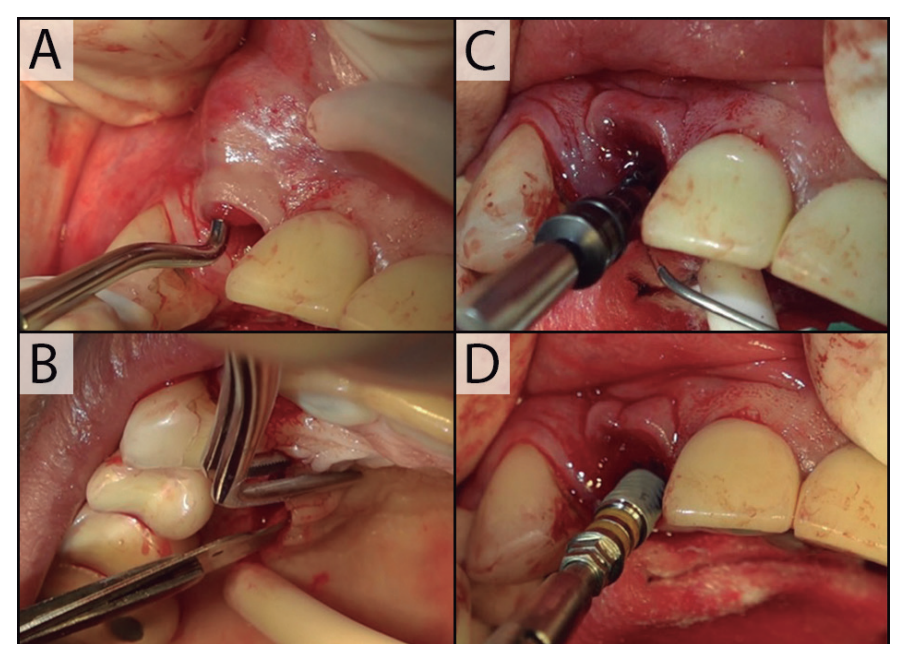

Figura 3. Inserción del nuevo implante. A colgajo tipo sobre conservando la integridad de las papilas y decolamiento de tejidos mediante instrumental específico para su posterior tracción coronal libre de tensión. B toma de injerto de tejido conectivo en bolsillo desde el paladar. C protocolo de fresado bajo irrigación continua de suero fisiológico. $D$ inserción del implante en posición protésicamente ideal.

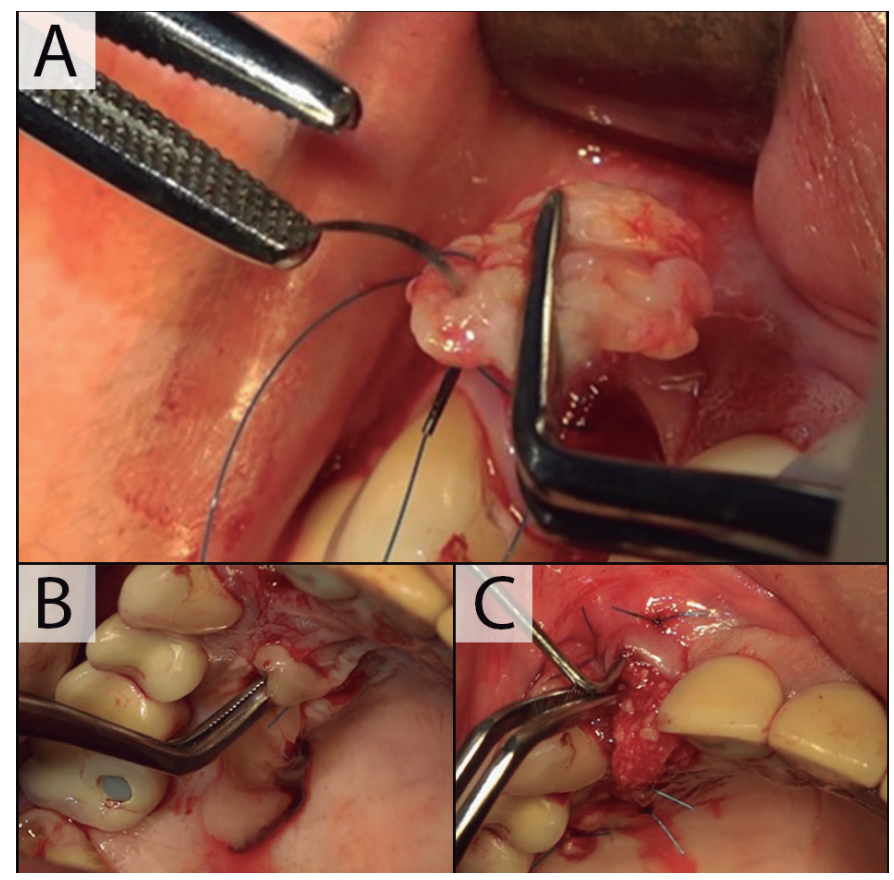

Figura 4. Técnica de regeneración ósea e injerto de tejido conectivo. A fijación del injerto de tejido conectivo mediante sutura. B cierre del segundo sitio quirúrgico en la zona palatina mediante membrana de L-PRF y sutura. C inserción del aloinjerto óseo mezclado con el centrifugado sanguíneo (sticky bone) en el compartimiento creado entre el implante y el injerto de tejido conectivo.

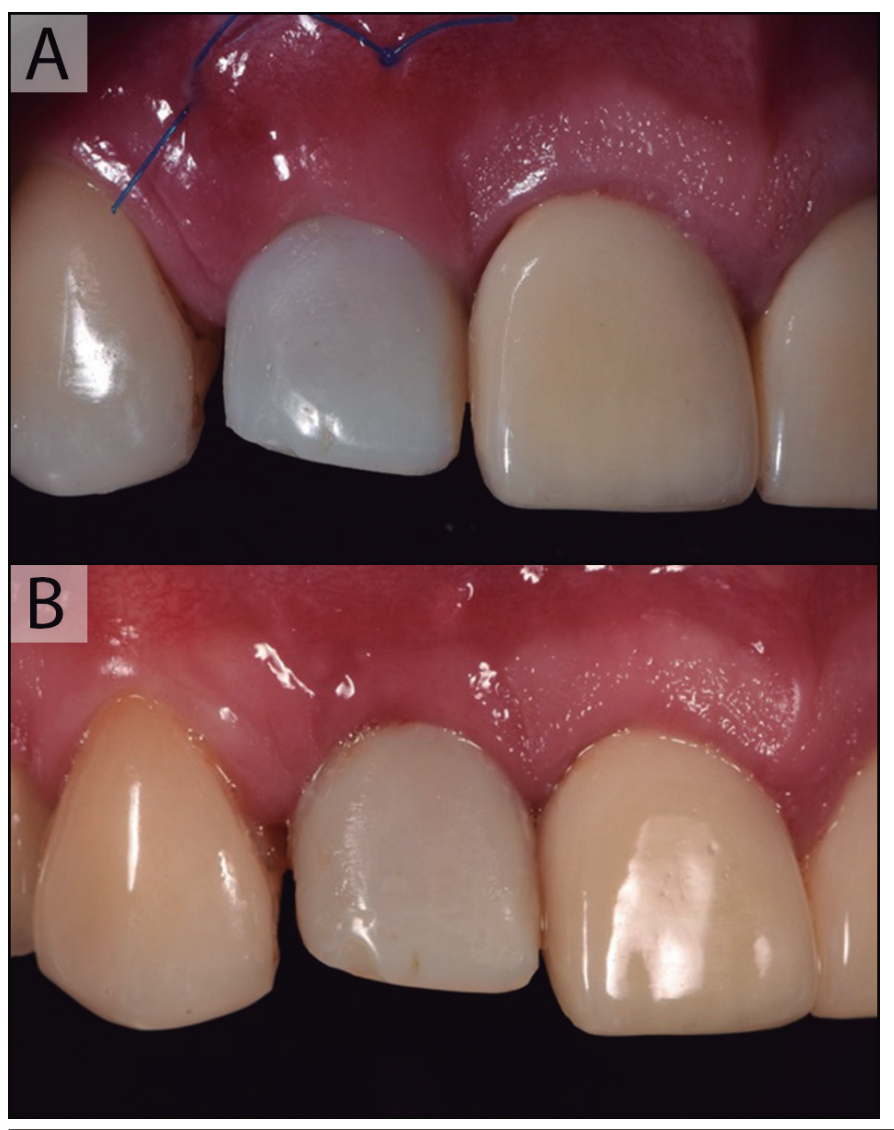

Figura 5. Provisionalización. A control a los 14 días. Se observa margen gingival en posición ideal. En el control a los 6 meses (B) se observa una leve migración apical de margen gingival, pero con tejidos estables y en salud.

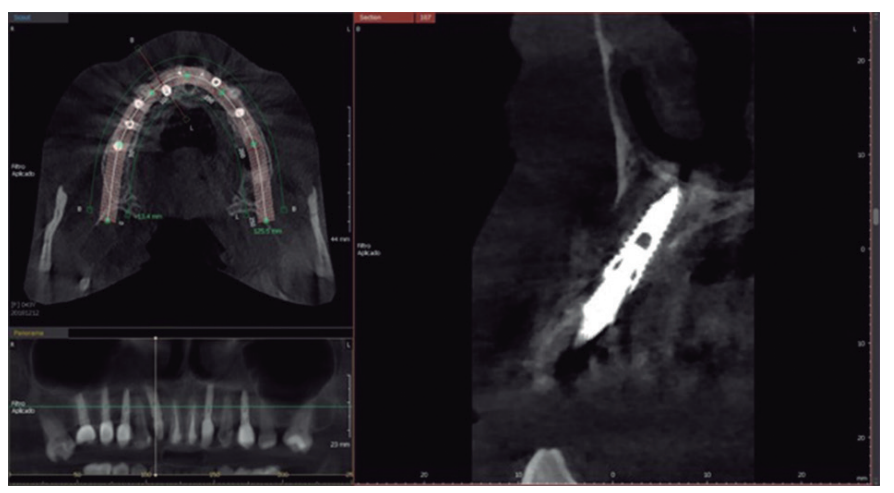

Figura 6. Cone beam de control post quirúrgico. Se observa el implante provisionalizado y la recuperación ósea en la zona vestibular. 


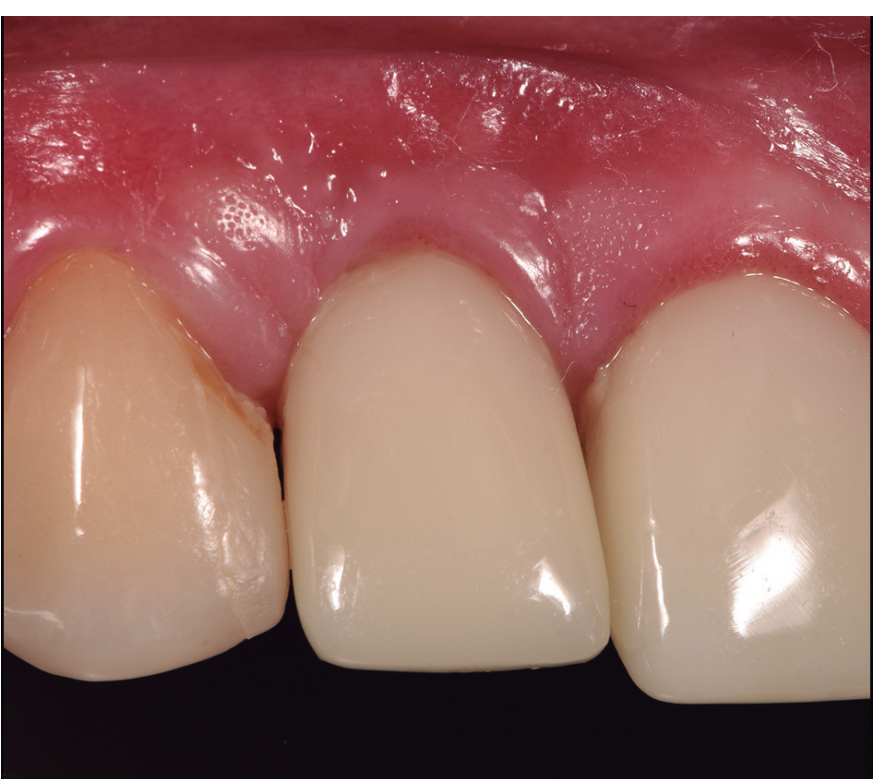

Figura 7. Control final post-cementación de la prótesis fija libre de metal. Se observa una posición levemente más alta del margen gingival con respecto a 1.1 y 1.3. La papila mesial está perfectamente conservada, mientras que la papila distal sufrió una leve retracción.

\section{DISCUSIÓN}

El gran desarrollo en biomateriales y técnicas de regeneración ósea guiada ha posibilitado un importante avance hacia el posicionamiento ideal de los implantes, guiado por la futura prótesis y no por la disponibilidad de hueso. De esta manera se ha logrado mejorar la predictibilidad y el éxito de los tratamientos rehabilitadores ${ }^{(8)}$. Sin embargo, la herencia dejada por las técnicas antiguas enfrenta diariamente al implantólogo con fracasos estéticos y biológicos, cuya resolución pasa en muchos casos por nuevas intervenciones quirúrgicas y el reposicionamiento de los implantes.

La mayoría de las técnicas descritas en la literatura para el abordaje de complicaciones estéticas, como la dehiscencia vestibular, presenta buenos resultados en términos de predictibilidad $(5,7,9-10)$. Recientemente, Zucchelli y cols. presentaron una clasificación de defectos de tejido blando periimplantario acompañada de recomendaciones para su abordaje ${ }^{(1)}$. Según esta clasificación, el caso reportado en este trabajo se consideraría tipo IV, encontrándose tanto el perfil de la corona como la cabeza del implante ubicados por fuera de la línea imaginaria que une los perfiles de los dientes adyacentes (Figura 1C). Si bien existía una adecuada conservación de las papilas (subclase a), la corrección mediante un tratamiento protésico se vio limitada por la presencia de una importante dehiscencia vestibular, lo que llevó a la decisión de realizar la explantación. A esto se sumó que el implante antiguo poseía una conexión hexagonal externa, las que presentan mayor micromovimiento del pilar y conllevan un mayor riesgo de complicaciones mecánicas y biológicas. Para este tipo de casos no existen protocolos establecidos con respecto a los tiempos de intervención ni a los procedimientos de regeneración. La utilización de un injerto óseo, acompañado de un injerto de tejido conectivo, buscaban por tanto, mejorar la predictibilidad estética y biológica del nuevo implante.

Si bien el resultado final de este caso es próximo al ideal, ya que se corrigió la posición del margen gingival, se recuperó la integridad de los tejidos blandos y se mejoró la apariencia estética de la restauración, al control final se observa una leve migración apical del margen gingival (Figura 7), acompañado de la pérdida de aproximadamente $1 \mathrm{~mm}$ de la papila distal. Lo primero se puede explicar en parte debido a la mayor profundidad de la técnica en bolsillo utilizada para extraer el injerto de la zona lateral del paladar, que, a diferencia de los injertos tomados desde la tuberosidad, posee un componente menor de lámina propia y un mayor contenido submucoso glandular y adiposo ${ }^{(13)}$. Por otra parte, si bien el uso de provisionalización inmediata ha demostrado disminuir la recesión gingival media entre 2.5 a 3 veces con respecto a una provisionalización tardía ${ }^{(14)}$, el éxito estético de la implantación inmediata con provisionalización inmediata se relaciona además a factores terapéuticos y del paciente ${ }^{(15)}$. En este caso, la falta de punto de contacto distal del provisorio (Figura $5 \mathrm{~A}$ ) pudo influir en la retracción de la papila distal.

Porúltimo, es interesante destacar que pese al corto tiempo de seguimiento del presente caso, es posible observar una buena predictibilidad mediante el protocolo combinado de una técnica conservadora de explantación mediante la llave de alto torque ${ }^{(11)}$, conservando el máximo de tejido óseo autólogo, y el uso de un injerto óseo enriquecido con elementos del plasma sanguíneo (sticky bone) ${ }^{(12)}$. La realización de ambas etapas en un solo tiempo quirúrgico permitió además un ahorro significativo de tiempo, sin sacrificar el resultado final del tratamiento. Es necesario, sin embargo, una observación a plazos mayores para constatar su éxito en el tiempo.

\section{CONCLUSIÓN}

La gran incidencia de defectos estéticos asociados a la mal posición de los implantes dentales hace imperante el desarrollo de protocolos para el manejo quirúrgico de estos casos. El éxito logrado mediante el protocolo combinado de explantación conservadora y regeneración tisular en el presente reporte entregan un primer lineamiento en esta dirección, cuya eficiencia y predictibilidad deben seguir siendo estudiadas.

\section{RELEVANCIA CLÍNICA}

En el presente reporte de caso se describe una técnica conservadora de explantación mediante llave de alto torque en combinación con técnicas de regeneración ósea e injerto de tejido conectivo. Se entrega de este modo al clínico una herramienta interesante para la resolución de casos críticos de mal posición de implantes asociados a defectos óseos y de tejido blando en zonas de alta demanda estética. A través del protocolo clínico propuesto se pretende sistematizar el tratamiento de estas complicaciones, mejorando su predictibilidad y éxito en el tiempo.

\section{CONFLICTO DE INTERESES}

Los autores del presente trabajo no declaran ningún conflicto de interés. El trabajo clínico realizado no contó con ningún tipo de financiamiento. El tratamiento fue cancelado íntegramente por el paciente.

\section{Bibliografía}

1. Zucchelli G, Tavelli L, Stefanini M, Barootchi S, Mazzotti C, Gori G, et al Classification of facial peri-implant soft tissue dehiscence/deficiencies at single implant sites in the esthetic zone. J Periodontol. 2019;90:1116-24.

2. De Bruyn H, Raes S, Matthys C, Cosyn J. The current use of patient-centered/ reported outcomes in implant dentistry: a systematic review. Clin Oral Implants Res. 2015;26:52-55

3. Gholami M. Mobilization of malpositioned dental implant using segmental osteotomy: A case report. J Stomatol Oral Maxillofac Surg. 2018;119:52-5.

4. Worni A, Marchand L, Sailer I, Cornish D, Hicklin SP. Explantation of an osseointegrated titanium implant using laser-induced thermo-necrosis: a case report. Int J Oral Maxillofac Implants. 2018;33:e151-e5.

5. Zucchelli G, Mazzotti C, Mounssif I, Mele M, Stefanini M, Montebugnoli L. A novel surgical-prosthetic approach for soft tissue dehiscence coverage around single implant. Clin Oral Implants Res. 2013;24:957-62.

6. Evans C, Chen S. Esthetic outcomes of immediate implant placements. Clin Oral Implants Res. 2008;19:73-80.

7. Mazzotti C, Stefanini M, Felice P, Bentivogli V, Mounssif I, Zucchelli G. Soft-tissue dehiscence coverage at peri-implant sites. Periodontol 2000. 2018;77:256-72

8. Testori T, Weinstein T, Scutella F, Wang HL, Zucchelli G. Implant placement in the esthetic area: criteria for positioning single and multiple implants. Periodontol 2000. 2018;77:176-96
9. Zucchelli G, Felice P, Mazzotti C, Marzadori M, Mounssif I, Monaco C, et al 5-year outcomes after coverage of soft tissue dehiscence around single implants: A prospective cohort study. Eur J Oral Implantol. 2018;11:215-24.

10. Burkhardt R, Joss A, Lang NP. Soft tissue dehiscence coverage around endosseous implants: a prospective cohort study. Clin Oral Implants Res. 2008;19:451-7.

11. Stajcic Z, Stojcev Stajcic LJ, Kalanovic M, Dinic A, Divekar N, Rodic M. Removal of dental implants: review of five different techniques. Int J Oral Maxillofac Surg. 2016;45:641-8

12. Sohn DS, Huang B, Kim J, Park WE, Park C. Utilization of autologous concentrated growth factors (CGF) enriched bone graft matrix (sticky bone) and CGF-enriched fibrin membrane in implant dentistry. J Implant Adv Clin Dent. 2015;7:11-29.

13. Sanz-Martin I, Rojo E, Maldonado E, Stroppa G, Nart J, Sanz M. Structural and histological differences between connective tissue grafts harvested from the lateral palatal mucosa or from the tuberosity area. Clin Oral Investig. 2019;23:957-64.

14. De Rouck T, Collys K, Wyn I, Cosyn J. Instant provisionalization of immediate single-tooth implants is essential to optimize esthetic treatment outcome. Clin Oral Implants Res. 2009;20:566-70.

15. Zucchelli G, Sharma P, Mounssif I. Esthetics in periodontics and implantology. Periodontol 2000. 2018;77:7-18. 